\title{
Miniscrews-assisted Lacebacks in Forsus to Minimize Lower Incisor Proclination
}

\author{
Dr Amit Prakash,' Dr Heena Kausar, ${ }^{2}$ Dr Sashi B Ekka³ \\ ${ }^{3}$ Reader, Department of Orthodontics, People's College of Dental Sciences, Bhopal, India \\ ¿Senior Lecturer, Vyas Dental College, Department of Orthodontics, Jodhpur, India
}

\section{ABSTRACT}

Forsus is a Class II corrector appliance used in growing as well as in young adults. Proclination and forward movement of lower incisor is the major drawback reported using this appliance. Forsus results in Class II correction mainly through dentoalveolar effect and minimal skeletal effect. We used a new innovation incorporating miniscrew assisted laceback to minimize lower incisor proclination. Utilization of miniscrew assisted laceback effectively reduces the unfavorable proclination and allows additional skeletal effects. This method applies a distal driving force on the lower anteriors, which minimizes lower incisor proclination. It is proven to be effective method in controlling the lower incisor flaring.

Key words: anchorage, Forsus, laceback, miniscrew

\section{INTRODUCTION}

Treatment of Class II malocclusion is always challenging as it involves basal jaw bone to which applying sound biomechanical principles execute the pave to achieve predictable result. Forsus appliance is applicable in sagittal advancement of the mandible without requiring patient's compliance as it can be worn in association with fixed appliance. ${ }^{1,2}$

Although many appliance systems that correct Class II malocclusion using dental anchorage exist; one common side effect is the proclination of lower incisors. Fixed functional appliances are associated with exertion of heavy orthodontic force on lower anterior and upper posterior teeth. The dentoalveolar changes make it highly significant both in maxillary and mandibular arches. Effects on mandible are mainly at the dentoalveolar level, with a large amount of mesial movement of the lower incisors. ${ }^{1,3}$
The conventional methods used to minimize these problems generally include: banding/bonding of second molar, lingual arch, cinching back of the mandibular archwire distal to the molars, use of mandibular rectangular archwires of greater size, addition of negative torque to the archwire in the lower incisor region etc. ${ }^{4-5}$ Thus, we have developed a new approach to minimize the lower incisor proclination.

\section{METHOD}

Angle's Class II Div 1 case with crowding case (Figure 1) was treated with 0.022-inch slot MBT brackets. Passive transpalatal arch and lingual arch were cemented to the maxillary and mandibular first molars respectively. Levelling and alignment proceeded until treatment reached a phase including $0.019 \times 0.025$-inch stainless steel archwires. Wires were cinched distal to the maxillary and mandibular first molars. Miniscrews were placed bilaterally in between second premolar and first
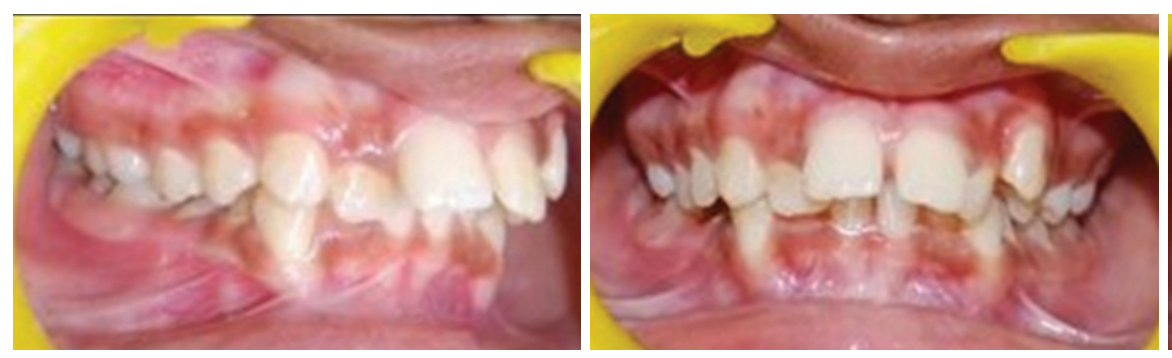

Figure 1: Pre-treatment photographs

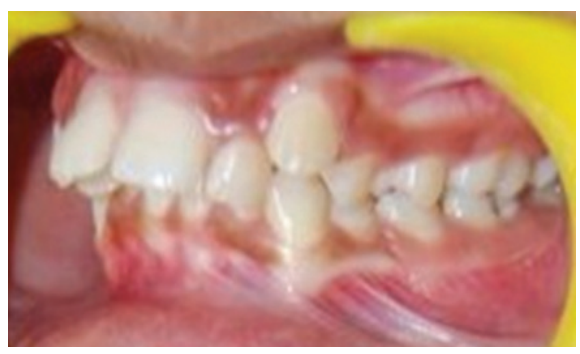



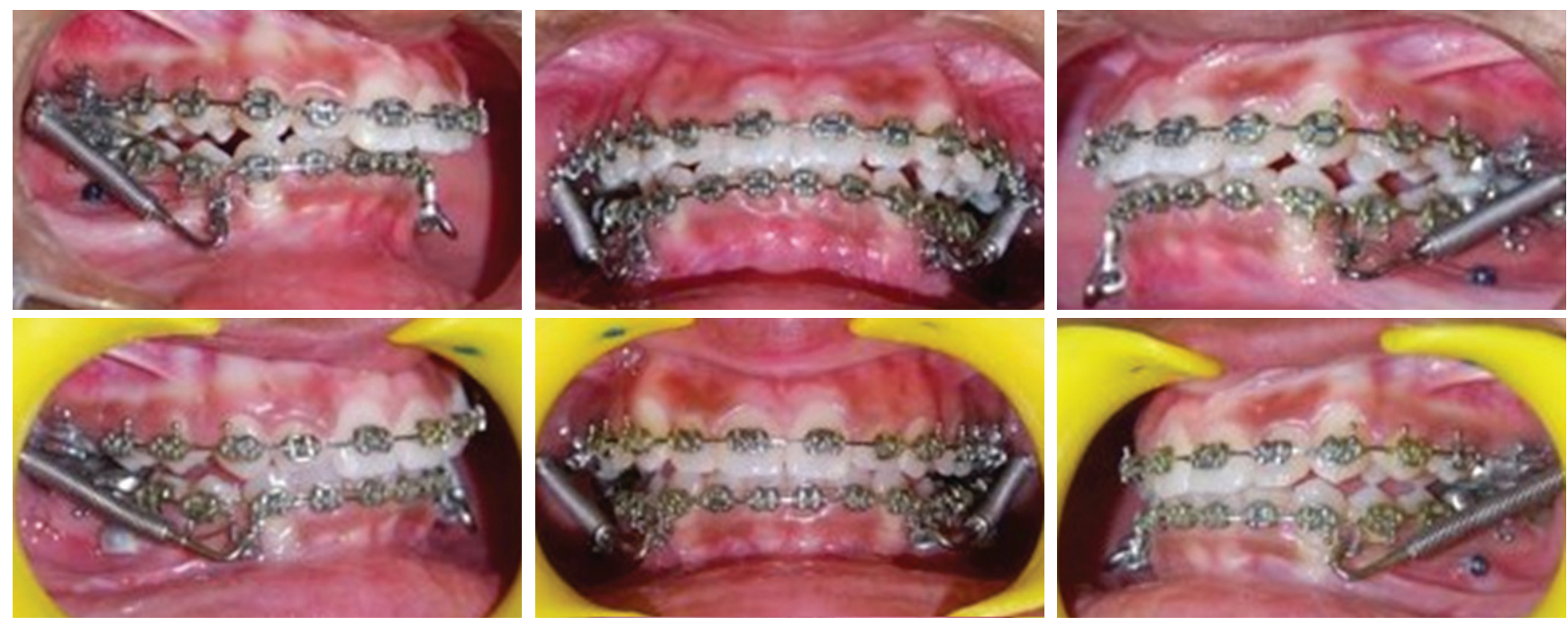

Figure 2: Forsus assisted lacebacks
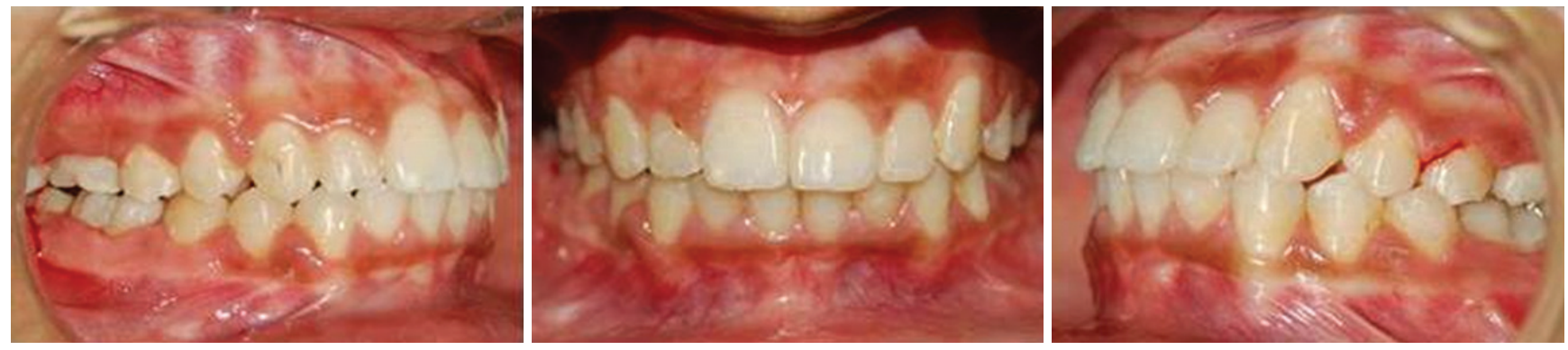

Figure 3: Post-treatment photographs

molar. Lacebacks were placed through the miniscrews to loops placed between canine and premolar (Figure 2). Size of the ligature wire used was 0.010 inch. Forces applied were parallel and near to the centre of resistance. Ligature wire attached to miniscrew applied a distal driving force on the lower anteriors, which minimized the lower incisor proclination. It also allowed maxillary arch distalization which added to mandibular arch correction. Less proclination of lower incisor achieved more skeletal changes in the patients (Figure 3).

\section{CONCLUSION}

The adverse effect of mandibular incisor flaring cannot be completely prevented by the use of conventional functional appliances; however it can be minimized by adding an extra anchorage in the lower arch. Proclination poses a problem in the stability of the occlusion and can be controlled with the help of miniscrews attached lacebacks effectively.

\section{OJN}

\section{REFERENCES}

1. Von Bremen J, Pancherz H. Efficiency of early and late Class II division 1 treatment. Am J Orthod Dentofacial Orthop. 2002;121:31-7.

2. Vogt W. The Forsus Fatigue Resistant Device. J Clin Orthod. 2006;40:368-77.

3. Jones G, Buschang PH, Kim KB, Oliver DR. Class II nonextraction patients treated with the Forsus Fatigue Resistant Device versus intermaxillary elastics. Angle Orthod 2008;78:332-8.

4. Pandis N, Polychronopoulou A, Sifakakis I, Makou M, Eliades T. Effects of levelling of the Curve of Spee on the inclination of mandibular incisors and expansion of dental arches: A prospective clinical trial. Aust Orthod J. 2010;26:61-5.

5. Aziz T, Nassar U, Flores-Mir C. Prediction of lower incisor inclination during X bow treatment based on initial cephalometric variables. Angle Orthod. 2012;82:472-9. 